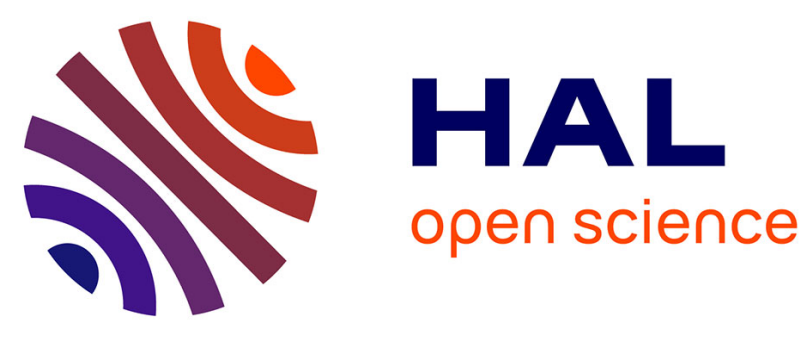

\title{
3D Chemical Shift-Encoded MRI for Volume and Composition Quantification of Abdominal Adipose Tissue During an Overfeeding Protocol in Healthy Volunteers
}

Angeline Nemeth, Berenice Segrestin, Benjamin Leporq, Kévin Seyssel, Khuram Faraz, Valerie Sauvinet, Emmanuel Disse, Pierre-Jean Valette, Martine Laville, Hélène Ratiney, et al.

\section{- To cite this version:}

Angeline Nemeth, Berenice Segrestin, Benjamin Leporq, Kévin Seyssel, Khuram Faraz, et al.. 3D Chemical Shift-Encoded MRI for Volume and Composition Quantification of Abdominal Adipose Tissue During an Overfeeding Protocol in Healthy Volunteers. Journal of Magnetic Resonance Imaging, 2019, 49 (6), pp.1587-1599. 10.1002/jmri.26532 . hal-01917470

\author{
HAL Id: hal-01917470 \\ https://hal.science/hal-01917470
}

Submitted on 13 Jul 2020

HAL is a multi-disciplinary open access archive for the deposit and dissemination of scientific research documents, whether they are published or not. The documents may come from teaching and research institutions in France or abroad, or from public or private research centers.
L'archive ouverte pluridisciplinaire HAL, est destinée au dépôt et à la diffusion de documents scientifiques de niveau recherche, publiés ou non, émanant des établissements d'enseignement et de recherche français ou étrangers, des laboratoires publics ou privés. 
1 Title: 3D chemical shift-encoded MRI for volume and composition

2 quantification of abdominal adipose tissue during an overfeeding

3 protocol in healthy volunteers

4 Author Names and Degrees:

5 Angeline Nemeth ${ }^{1}$, MS, Bérénice Segrestin ${ }^{2,3}$, MD, Benjamin Leporq ${ }^{1}, \mathrm{PhD}$, Kevin Seyssel ${ }^{4}$,

$6 \mathrm{PhD}$, Khuram Faraz ${ }^{1}, \mathrm{PhD}$, Valérie Sauvinet ${ }^{2}$, MS, Emmanuel Disse ${ }^{2,3}$, MD-PhD, Pierre-Jean

7 Valette $^{5}, \mathrm{MD}-\mathrm{PhD}$, Martine Laville ${ }^{2,3}, \mathrm{MD}-\mathrm{PhD}$, Hélène Ratiney ${ }^{1}, \mathrm{PhD}$, and Olivier Beuf ${ }^{1}$,

$8 \mathrm{PhD}$

\section{Author Affiliations:}

$10 \quad{ }^{1}$ Univ Lyon, INSA-Lyon, Université Claude Bernard Lyon 1, UJM-Saint Etienne, CNRS,

11 Inserm, CREATIS UMR 5220, U1206, F69621, Lyon, France, ${ }^{2}$ Centre de Recherche en

12 Nutrition Humaine Rhône-Alpes (CRNH-RA), Centre Hospitalier Lyon Sud, Pierre-Bénite,

13 Lyon, France, ${ }^{3}$ Institut National de la Santé et de la Recherche Médicale Unit 1060, CarMeN

14 Laboratory, Lyon 1 University, Oullins, France, ${ }^{4}$ Department of Physiology, Faculty of

15 Biology and Medicine, University of Lausanne, Lausanne, Switzerland, ${ }^{5}$ Hospices Civils de

16 Lyon, Département d'imagerie digestive, CHU Edouard Herriot, Lyon, France

\section{Correspondence to:}

18 Olivier Beuf, Univ Lyon, INSA-Lyon, Université Claude Bernard Lyon 1, UJM-Saint

19 Etienne, CNRS, Inserm, CREATIS UMR 5220, U1206, F69621, Lyon, France

20 Email: Olivier.beuf@creatis.insa-lyon.fr 


\section{Grant Support:}

22 LABEX PRIMES (ANR-11-LABX-0063), the "Investissements d'Avenir" program ANR-11-

23 IDEX-0007, IHU OpéRa (ANR-10-IBHU-0004).

24 Running Title: Quantification of adipose tissue by CSE-MRI 


\section{Abstract}

\section{BACKGROUND}

Overweight and obesity are a major worldwide health concern characterized by an abnormal accumulation of fat in adipose tissue (AT) and liver.

30

\section{PURPOSE}

To evaluate the volume and the fatty acid (FA) composition of the subcutaneous adipose tissue (SAT) and the visceral adipose tissue (VAT) and the fat content in the liver from 3D CSE-MRI acquisition, before and after a 31 days overfeeding protocol.

\section{STUDY TYPE}

Prospective and longitudinal study.

\section{SUBJECTS}

21 non-obese healthy male volunteers

\section{FIELD STRENGTH/SEQUENCE}

A 3D spoiled-gradient multiple echo sequence and STEAM sequence were performed at 3T.

\section{ASSESSMENT}

AT volume was automatically segmented on CSE-MRI between L2 to L4 lumbar vertebrae and compared to the DEXA measurement. CSE-MRI and MRS data were analyzed to assess

3 the proton density fat fraction (PDFF) in the liver and the FA composition in SAT and VAT.

4 Gas chromatography-mass spectrometry (GC-MS) analyses were performed on 13 SAT samples as a FA composition countermeasure. 
47 Paired t-test, Pearson's correlation coefficient and Bland Altman plot were used to compare

48 measurements.

49

\section{RESULTS}

SAT and VAT volumes significantly increased $(\mathrm{p}<0.001)$. CSE-MRI and DEXA measurements were strongly correlated $(r=0.98, \mathrm{p}<0.001)$. PDFF significantly increased in the liver $(+1.35, \mathrm{p}=0.002$ for CSE-MRI, $+1.74, \mathrm{p}=0.002$ for MRS $)$. FA composition of SAT and VAT appeared to be consistent between localized-MRS and CSE-MRI (on whole segmented volume) measurements. Significant difference between SAT and VAT FA composition was found ( $\mathrm{p}<0.001$ for CSE-MRI, $\mathrm{p}=0.001$ for MRS). MRS and CSE-MRI measurements of the FA composition were correlated with the GC-MS results (for ndb: $\mathrm{r}_{\text {MRS/GC-MS }}=0.83 \mathrm{p}<0.001, \mathrm{r}_{\mathrm{CSE}-}$ MR/GC-MS $=0.84, p=0.001 ;$ for nmidb: $\mathrm{r}_{\text {MRS/GC-MS }}=0.74, \mathrm{p}=0.006, \mathrm{r}_{\mathrm{CSE}-\mathrm{MR} / \mathrm{GC}-\mathrm{MS}}=0.66, \mathrm{p}=0.020$ )

\section{DATA CONCLUSION}

The follow-up of liver PDFF, volume and FA composition of AT during an overfeeding diet was demonstrated through different methods. The CSE-MRI sequence associated with a dedicated post-processing was found reliable for such quantification.

Key words (3 to 6): Fatty acid composition, in vivo, MR spectroscopy, chemical shiftencoded imaging, overfeeding, gas chromatography-mass spectrometry 


\section{Terminology}

66 - AT, adipose tissue

67 - FA, fatty acid;

68 - SFA, saturated fatty acid;

69 - MUFA, monounsaturated fatty acid;

70 - PUFA, polyunsaturated fatty acid;

71 - $n d b$, number of double bonds;

72 - nmidb, number of methylene-interrupted double bonds;

73 - $\mathrm{SFA}_{\text {indx }}$, proportion of saturated fatty acid estimated by $n d b$ and $n m i d b$;

74 - $\mathrm{MUFA}_{\text {indx }}$, proportion of monounsaturated fatty acid estimated by $n d b$ and $n m i d b$;

75 - PUFA $_{\text {indx }}$, proportion of polyunsaturated fatty acid estimated by $n d b$ and $n m i d b$;

76 - CL, chain length;

77 - SAT, subcutaneous abdominal adipose tissue;

78 - VAT, visceral adipose tissue;

79 - PDFF, proton density fat fraction

80 - CSE-MRI, chemical shift-encoded magnetic resonance imaging

81 - CV, coefficient of variation

82 - GC-MS, gas chromatography-mass spectrometry

83 - IFCC, International Federation of Clinical Chemistry

84 - TAG, triacylglycerol

85 - SNR, signal to noise ratio 


\section{Text (5199words):}

\section{INTRODUCTION}

Obesity is a complex disease combining genetic factors still poorly identified and environmental factors mainly related to diet and physical inactivity(1). Overweight and obesity are major worldwide health issues increasing the risk to develop pathologies such as diabetes, cardiovascular disease and Non Alcoholic Fatty Liver Diseases (NAFLD). Although obesity is defined by a body mass index (BMI) greater than or equal to $30 \mathrm{~kg} / \mathrm{m}^{2}$ for humans, distribution of adipose tissue is decisive in the assessment of cardio-metabolic risk factors(2). People with abdominal fat accumulation have commonly an altered metabolic profile (also called metabolic syndrome). The expansion of visceral adipose tissue (VAT), leading to an increase in waist circumference, is associated with a higher risk to develop insulin resistance and type 2 diabetes unlike the expansion of subcutaneous adipose tissue (SAT) (3). BMI is not relevant to predict these risks because no distinction is made between fat mass and lean mass. Dual-energy X-ray absorptiometry (DEXA) allows measurement and distribution of body fat. To be more accurate, some imaging modalities like Magnetic Resonance Imaging (MRI) and computed tomography (CT) (4) can assess independently the distribution of SAT and VAT unlike DEXA. The major drawback of CT is the ionizing radiation exposure of patients whereas MRI allows the measurement of fat volumes without any known short- or long-term side effects. Over the last decades, various segmentation methods of SAT and VAT volume have emerged based on various MRI acquisition such as T1-weighted, T2-weighted or chemical-shift encoded imaging (CSE-MRI), each one with advantages and disadvantages (5). T1-weighted and T2-weighted image contrast varies with B0 field strength which can be a problem in the reproducibility of the results in multi-center studies. CSE-MRI can give an accurate estimate of proton density fat fraction (PDFF) which is a biomarker (6). Beyond volume quantification, the lipid composition of these tissues could be a relevant biomarker 
113 (7).When analyzed by mass spectrometry (8), fatty acid composition (FA) of AT has been

114 associated with AT function (adipogenesis, differentiation, lipid metabolism) with large

115 differences between SAT and VAT, shedding light on the pathophysiology of metabolic

116 diseases $(8,9)$. This analysis requires access to human tissue, and cannot be widely proposed.

117 Surrogate markers of lipid composition of AT are needed. Magnetic Resonance Spectroscopy

118 (MRS) is able to provide non-invasively the proportions of saturated (SFA), monounsaturated

119 (MUFA) or polyunsaturated (PUFA) fatty acids. Recent studies (10-12) demonstrated the

120 feasibility of assessing the composition of fat based on multiple gradient echo imaging also

121 named chemical shift-encoded MRI (CSE-MRI). Studies focusing on liver diseases have

122 extensively compared the MRS and MRI methods for the quantification of the PDFF(13-15).

123 The aim of this work was to assess CSE-MRI method to detect content and composition

124 changes of fat storage in healthy volunteers during a 31 days overfeeding protocol while

125 comparing CSE-MRI results with DEXA, MRS and GC-MS measurements.

\section{MATERIALS AND METHODS}

\section{Overfeeding Protocol}

128 From February 2014 to December 2016, volunteers were recruited via notice boards and

129 electronic bulletins. To be eligible, participants had to be a man aged between 18 and 55 years

130 with a BMI between $23.0 \mathrm{~kg} / \mathrm{m}^{2}$ and $27.0 \mathrm{~kg} / \mathrm{m}^{2}$ and low physical activity. Volunteers were

131 excluded if they had personal medical history of type 1 or 2 diabetes. Written informed

132 consent was obtained from all the subjects. All men underwent 31 days of high-sucrose and

133 high-fat overfeeding by eating the equivalent of $+50 \%$ of their daily energy requirements

134 while maintaining their eating and sport activity habits. This protocol was validated by an

135 ethics committee and was registered on the clinicaltrials.gov site (NCTXXXXXXXX). Only

136 results from subjects with a placebo complement to their eating are reported in this paper. 
137

138

139

140

141

142

143

144

145

146

147

148

149

150

151

152

153

154 close to the defined region in MRI method.

155

156 Subjects underwent two STEAM sequences in a row for test-retest using respiratory 157 triggering with voxel placed in liver, VAT and SAT tissues (parameters in the caption of 158 Figure 1). This protocol was performed at the baseline examination (MRS1) and repeated 159 after the overfeeding (MRS2). 
160

161

162

163

164

165

166

167

168 182 fused-silica column (SP2380, $60 \mathrm{~m}$ x $0.20 \mathrm{~mm}$ x $0.25 \mu \mathrm{m}$ film thickness; Supelco). Injection

$183(1 \mu \mathrm{L})$ was performed in splitless mode at $240{ }^{\circ} \mathrm{C}$. FAs were separated with the following

\section{FA Analysis By Gas Chromatography-Mass Spectrometry (GC-MS)}

The total FA compositions of the SAT samples were determined independently by GC-MS. FAs profiles were obtained from the triacylglycerol (TAG) fraction which is representative of the intracellular content of the SAT.

\section{Sample Collect}

A needle biopsy of abdominal SAT was performed $10 \mathrm{~cm}$ from the umbilicus, under local anesthesia (1\% lidocaine, BD microlance $21 \mathrm{G} 1^{1 / 2}$ inch, $0.8 * 40 \mathrm{~mm}$ needles), for $300 \mathrm{mg}$ to $1 \mathrm{~g}$ adipose tissue samples. Subsequent biopsy was taken contralaterally. Fat samples were immediately frozen in liquid nitrogen and stored at $-70{ }^{\circ} \mathrm{C}$.

\section{Sample Preparation}

Approx. $50 \mathrm{mg}$ cryogenically crushed sample were weighed exactly before proceeding to the total lipid extraction using $6 \mathrm{~mL}$ of a mixture of chloroform/methanol (2:1, vol:vol) according to the Folch method (17). An internal standard (TAG C17:0, glycerol triheptadecanoate), corresponding to $60 \mu \mathrm{g}$ of $\mathrm{C} 17: 0 / \mathrm{mg}$ of tissue, was added in order to quantify the FAs occurring from the TAG fraction. The TAG fraction was then obtained from 1/40e of the total lipid extract by thin-layer chromatography on silica-gel plates with a mobile phase of hexane: diethyl ether:acetic acid (80:20:1, vol:vol:vol) and was submitted to direct methylation according to a modification of the technique described elsewhere (18).

\section{GC-MS Analysis}

The amounts of FAs were measured by GC/EI-MS (EI: electron ionization) using a quadrupole mass spectrometer (model MS 5975, Agilent Technologies, Massy, France) connected to a gas chromatograph (model GC6890, Agilent Technologies) equipped with a 
184 oven program: (a) $50{ }^{\circ} \mathrm{C}$ for $1 \mathrm{~min}$; (b) increase at a rate of $20{ }^{\circ} \mathrm{C} \cdot \mathrm{min}^{-1}$ to $175^{\circ} \mathrm{C}$ and hold for

$1859.75 \mathrm{~min}$; (c) increase at a rate of $2{ }^{\circ} \mathrm{C} . \min -1$ to $217{ }^{\circ} \mathrm{C}$ and hold at $217{ }^{\circ} \mathrm{C}$ for $1 \mathrm{~min}$. Mass

186 spectra were obtained from the Total Ion Chromatogram (TIC) over a mass range of m/z 35187450.

Comparison With NMR Methods

189 In order to obtain comparable results between the GC-MS and NMR methodologies, the mean 190 number of double bound $\left(n d b_{G C}\right)$ and the mean of methylene-interrupted double bound

$191 \quad\left(\right.$ nmidb $\left._{G C}\right)$ by triglyceride chain were computed as:

$$
\begin{aligned}
n d b_{G C} & =\left(\sum_{i}^{N} n d b_{i} * p_{i}\right) * 3 \\
n m i d b_{G C} & =\left(\sum_{i}^{N} n m i d b_{i} * p_{i}\right) * 3 \\
C L_{G C} & =\left(\sum_{i}^{N} C L_{i} * p_{i}\right)
\end{aligned}
$$

192 Where $N$ was the number of the different type of FAs analyzed, $n d b_{i}$ number of double bound

193 of the $\mathrm{i}^{\text {th }} \mathrm{FA}$, nmidb $_{i}$ number of methylene-interrupted double bound of the $\mathrm{i}^{\text {th }} \mathrm{FA}, C L_{i}$ the number of carbon in FA chain and $p_{i}$ the relative proportion of the $i^{\text {th }}$ FA.

196 For spectrum acquired in SAT, similarly to the eddy current correction performed usually using the water phase signal, the time dependent phase variation of the signal of the first echo ( $\mathrm{TE}=14 \mathrm{~ms})$ was corrected with the time dependent phase of the second echo $(\mathrm{TE}=24 \mathrm{~ms})$ which was less impacted by eddy current effects. This correction was possible because 1) the methylene $-\mathrm{CH}_{2 \mathrm{n}^{-}}$amplitude peak was the unique preponderant component, and 2) $\Delta \mathrm{TE}=\mathrm{TE}_{2}-$ 
$\mathrm{TE}_{1}$ was small compared to the lipid $\mathrm{T} 2$ and $1 / \mathrm{J}(4<\mathrm{J}<8 \mathrm{~Hz}$, for in vivo FA spectral). This correction needs exactly the same frequency content in the two spectra. So it was not applied to the spectra from the VAT tissue because of the presence of a low water peak in some case. In this latter case, the signal is probably altered due to peristalsis of the digestive tract from one echo to the other which could explain why the water peak contribution varies from one echo to the other. The quantification method, described shortly below and in (19), was applied only on the spectrum of the first echo; the other echoes were acquired to estimate the $\mathrm{T} 2 \mathrm{k}$, i.e the apparent $\mathrm{T} 2$ of each resonating lipid component $(\mathrm{k} \in[1, \ldots, 10]) . \mathrm{T} 2_{\mathrm{k}}$ estimations were made in the frequency domain, for each resonance, with a nonlinear least-square estimation of the mono-exponential $\mathrm{S}_{\mathrm{k}}(\mathrm{TE})=\mathrm{S} 0_{\mathrm{k}} * \exp \left(-\mathrm{TE} / \mathrm{T} 2_{\mathrm{k}}\right)$, where $\mathrm{S}_{\mathrm{k}}(\mathrm{TE})$ was the measured integral of the $\mathrm{k}^{\text {th }}$ peak at TE (6 echoes varying regularly from $14 \mathrm{~ms}$ to $64 \mathrm{~ms}$ by step of $10 \mathrm{~ms}), \mathrm{S} 0_{\mathrm{k}}$ the amplitude at $\mathrm{TE}=0$ and $\mathrm{T} 22_{\mathrm{k}}$ the $\mathrm{T} 2$ of the $\mathrm{k}^{\text {th }}$ peak.

To quantify the PDFF in the liver, a Voigt (20) model with 2 peaks was applied on the spectrum to estimate the amplitude of the water peak at $4.7 \mathrm{ppm}$ and the main peak of fat at $1.3 \mathrm{ppm}$. The PDFF was then computed by $\mathrm{PDFF}=\mathrm{A}_{\mathrm{f}} /\left(\mathrm{A}_{\mathrm{w}}+\mathrm{A}_{\mathrm{f}}\right)$. The non-linear least square optimization of the Voigt model was applied on the 200 first points of the FID ( 100ms and omitting the first point).

The MRS results reported in the rest of the paper are the mean quantification values of the two consecutive acquisitions.

\section{CSE-MRI Processing And Analysis}

\section{Automated Segmentation Method Of SAT And VAT}

Automatic segmentation was simultaneously performed exploiting the PDFF mapping described in (21) as well as the first and second echo images (out of phase and in phase respectively) to separate SAT from VAT. An operator determined the slices corresponding to 
the upper of L2 and the lower of L4. A first reference slice chosen by the user in the stack of images was segmented automatically. Manual correction can be done if necessary. Thanks to 3D acquisition and low partial volume effect, the mask of a slice (reference slice for the beginning) was used as a priori knowledge for the determination of the next slice mask using the Lankton level set algorithm (22). After segmentation, two binary masks were created one for SAT and the other for VAT and multiplied to the PDFF map PDFF $_{\mathrm{SAT}}$ and PDFF $\mathrm{PAT}_{\mathrm{V}}$ map). Only pixels with a PDFF higher than $55 \%$ were kept in order to remove vertebrae and intramuscular adipose tissue contributions to VAT volume. In some regions of large static field inhomogeneity, at the level of iliac crests, fat/water swaps led to erroneous PDFF contribution in particular in SAT. In this case, a manual correction was made.

\section{SAT, VAT And TAT Volumes}

The SAT and VAT masks were binary images. The total abdominal adipose tissue (TAT) was calculated by adding the SAT and VAT masks. Adipose tissue volumes were calculated by counting all pixels in the mask of SAT (or VAT or TAT) considering that each pixel represented $100 \%$ of fat and multiplying by the elementary volume (voxel of $9.8410^{-3} \mathrm{~cm}^{3}$ ). The total abdominal adipose tissue (TAT) volume delimited by the L2 and L4 vertebrae was converted into mass using the assumed an average density of $0.92 \mathrm{~g} / \mathrm{cm}^{3}(23)$ and compared to the DEXA measurement on android region. The total fat content in android region considering the PDFF of the tissues (adipose tissues, muscles and vertebrae) was also analyzed and computed as the sum of each element of the matrix defined by $\left(\mathrm{PDFF}_{\mathrm{SAT}}+\mathrm{PDFF}_{\mathrm{VAT}}\right) * 9.8410^{-3} \mathrm{~cm}^{3} * 0.92 \mathrm{~g} / \mathrm{cm}^{3}$.

\section{Fat Content In The Liver}

The region of interest (ROI) in the liver was manually defined on a unique slice on which the right hepatic portal vein appeared the largest in axial plane. The mean value of PDFF in this ROI was computed. To compare CSE-MRI and MRS measurements, the mean PDFF value 
250

251

252

253

254

was computed in an ROI corresponding to the MRS voxel size and labeled "localized CSEMRI measurement". This ROI was located automatically on the CSE-MRI data using MRS voxel coordinates. MRI was acquired in breath-holding whereas MRS was in free breathing. This difference led to localization errors of ROI on CSE-MRI. Thus, a manual ROI repositioning was visually performed in the liver.

\section{Calculation Of FA Composition (CSE-MRI And MRS)}

To avoid any difference linked to data processing, the FA quantification method used the same model function and implementation for CSE-MRI (10) and MRS (19). Indeed, in this previous publication (19), it has been shown that, despite different J-evolutions between STEAM sequence and 3D CSE-MRI, the model used in CSE-MRI can be used for MRS data analysis and lead to consistent results. FA composition was determined by a fitting procedure applied in the time domain on specific points defined by the methylene chemical shift: $\mathrm{t}=$ $\mathrm{t}=\mathrm{n}^{*} \mathrm{t}_{\mathrm{e}}$ with $\mathrm{t}_{\mathrm{e}}=\pi /\left((4.7-1.3) * \mathrm{~B}_{0} * \gamma\right)=1.15 \mathrm{~ms}$ and $\mathrm{n}=1, \ldots, 32$ for MRS processing and $\mathrm{n}=1, \ldots, 8$ for CSE-MRI. The model function used was defined as:

$f(t)=\operatorname{real}\left(\left(\mathrm{Aw} * n_{\text {water }} * e^{-\frac{(T E+t)}{T 2_{w}}}+A f * \sum_{k=1}^{8}\left(n_{k}(n d b, n m i d b, C L) *\right.\right.\right.$ $\left.\left.e^{2 \pi i \Delta f_{k} t} * e^{-\frac{(T E+t)}{T 2_{k}}}\right)\right) * e^{-\frac{t}{T 2^{\prime}}}$

where Aw and Af represent the numbers of water and triglycerides molecules respectively, $\mathrm{n}_{\mathrm{k}}(\mathrm{ndb}, \mathrm{CL}, \mathrm{nmidb})$ the number of protons in the fat spectrum component $\mathrm{k}$ in terms of ndb, CL and nmidb (Table 1), $f_{k}$ the frequency shift between water and each fat spectrum component $\mathrm{k}, \mathrm{n}_{\mathrm{water}}$ the number of protons in a water molecule, $\mathrm{T} 2_{\mathrm{k}}$ the transverse relaxation of the $\mathrm{k}^{\text {th }}$ peak, $\mathrm{T} 2_{\mathrm{w}}$ the transverse relaxation of the water peak, and the $\mathrm{T} 2$ ' the global 
272 relaxation time decay induced by $\mathrm{B} 0$ dispersion. For MRS processing, the $\mathrm{T} 2 \mathrm{k}_{\mathrm{k}}$-values were

273 estimated before the fitting and T2' was estimated during the processing (TE $=14 \mathrm{~ms}$ ). For

274 CSE-MRI processing, the global apparent T2* $(1 / \mathrm{T} 2 *=1 / \mathrm{T} 2+1 / \mathrm{T} 2$ ') value was computed (TE

$275=0 \mathrm{~ms})$. FA composition was derived from ndb and nmidb with the following relations (11):

$$
\begin{gathered}
P U F A_{\text {indx }}=\frac{n m i d b}{3} * 100 \\
M U F A_{\text {indx }}=\frac{(n d b-2 * n m i d b)}{3} * 100 \\
S F A_{\text {indx }}=100-P U F A_{\text {indx }}-M U F A_{\text {ind }}
\end{gathered}
$$

276 PUFA $_{\text {indx }}$ is the relative proportion of polyunsaturated FA, MUFA $A_{\text {indx }}$ the relative proportion 277 of mono-unsaturated FA and $\mathrm{SFA}_{\text {indx }}$ the relative proportion of saturated FA.

278 The CSE-MRI measurement of PUFA indx $_{\text {(respectively MUFA }}$ indx and SFA indx $_{\text {) was calculated }}$ 279 on whole-segmented volume of SAT (respectively VAT). To see the influence of the 280 inhomogeneity of the B0 field, another calculation was also computed excluding pixels at the 281 boundary between air/water or fat interface and pixels with an estimation of $\mathrm{T} 2 *$ under $10 \mathrm{~ms}$ 282 (figure 1 in supplementary material).

\section{Statistical Analysis}

284 Anthropometric measurements are expressed as mean \pm SEM. Pearson's correlation coefficient and Bland Altman plot were used to compare reference measurements (DEXA or MRS) and CSE-MRI measurements. Coefficient of variation (CV) was computed to analyze

287 the variability of the measurements. Paired t-tests were performed to compare data from 288 MRI1 (or MRS1) and MRI2 (or MRS2) for each quantified parameters (PDFF in the liver, 289 volume and FA composition of AT). The normality of each data distribution was tested using the Shapiro-Wilk test. In the case of non-normal distribution, a paired sample Wilcoxon rank 
291 test (non-parametric test) was performed in addition to the t-test. P-value <0.05 was 292 considered as significant. Statistical analyses were made with the software OriginPro 8.5.1 293 SR2 (OriginLab Corporation, Northampton, England).

\section{RESULTS}

\section{Subjects}

296 Baseline characteristics of 21 non-obese healthy male volunteers are summarized on Table 2. 297 An MRI examination after overfeeding could not be performed for one subject and the MRS 298 acquisitions from another subject before overfeeding were missing.

299

\section{Volumes}

\section{MRI vs DEXA Before Intervention}

On a total of 21 subjects, 18 underwent DEXA scanning on the same device (DEXA, Lunar Prodigy GE Medical Systems) and three on a different one (Discovery A, Hologic, Bedford, MA, USA). Only data from the first 18 subjects were used for the comparison. An excellent correlation between CSE-MRI measurement of TAT and DEXA measurement of android region $(\mathrm{r}=0.98, \mathrm{p}<0.001)$ was found with a constant lower value of MRI-based estimate (bias of $-120 \mathrm{~g}$, Bland-Altman plot Figure 2). This bias represented $6 \%$ in average of the android region mass. The total fat content measured by CSE-MRI considering PDFF of tissues was also highly correlated to DEXA measurement of android region $(r=0.94, p<0.001$, bias of $+96 \mathrm{~g})$.

\section{After The Overfeeding Intervention}

The subjects gained $2.5 \pm 0.3 \mathrm{~kg}$, with a $1.6 \pm 1.1 \mathrm{~kg}$ total fat mass gain measured by DEXA (Table 2). SAT, VAT and TAT volumes quantified using CSE-MRI sequence significantly increased between the two examinations $\left(+91 \mathrm{~cm}^{3}, \mathrm{p}<0.001 ;+129 \mathrm{~cm}^{3}, \mathrm{p}<0.001 ;+219 \mathrm{~cm}^{3}\right.$, $\mathrm{p}<0.001$ respectively). A significant increase in fat mass measured by DEXA in android 
315 region was also observed $(+191 \mathrm{~g}, \mathrm{p}=0.001)$ and was correlated to the CSE-MRI

316 measurements of TAT $(\mathrm{r}=0.80, \mathrm{p}<0.001, \mathrm{n}=16)$. The ratio VAT/SAT also significantly

317 increased after overfeeding $(+0.06, \mathrm{p}=0.020)$.

\section{$318 \quad$ Fat Content In Ihe Liver}

319 Hepatic fat content is low in healthy subjects and difficult to detect. In some MRS 320 measurements, fat content could not be detected: 2/20 at the baseline (MRS1). Over the total 321 of 21 subjects at baseline, one subject presented an abnormal level of PDFF in the liver 322 (PDFF $>10 \%$ ) based on CSE-MRI measurements (Figure 3a). Surprisingly, its corresponding 323 MRS measurement indicated a normal level of PDFF (PDFF=3.33\%). This subject had a non324 homogeneous (figure 2 in supplementary material) fat content in the liver and the MRS 325 measurement was made in a low-fat region of the liver.

327 At baseline, the correlation between localized CSE-MRI and MRS measurements was poor $\left(\mathrm{r}^{2}\right.$ $328=0.50, \mathrm{y}=1.02 \mathrm{x}+0.57)$. In Addition, the localized CSE-MRI gave larger PDFF-values $(+0.60$ 329 percentage points). After the overfeeding, the correlation was improved $\left(\mathrm{r}^{2}=0.80\right.$, $330 \mathrm{y}=1.04 \mathrm{x}+0.42)$ with still a lager value of CSE-MRI PDFF measurements $(+0.55$ percentage 331 points). Interestingly, even if the CSE-MRI seemed to give larger PDFF-values in the case of 332 low-fat content, variations of PDFF between the two examinations measured by MRS or 333 CSE-MRI were found to be very close $\left(r^{2}=0.77, y=0.92 x+0.09\right)$. Moreover, the bias between 334 these two measurements was small (larger value of localized CSE-MRI +0.05 percentage 335 points, Bland-Altman Figure 4). The Figure 4 shows an outlier point with a $\Delta$ MRS (MRS2336 MRS1) PDFF value at 3.37 and a $\triangle$ MRI (MRI2-MRI1) PDFF value at 0.39 . We must notify 337 that the two subsequent MRS2 acquisitions of the subject gave significantly $(\mathrm{p}<0.001)$ 338 different results: $6.32 \%$ at the first acquisition and $2.46 \%$ at the second acquisition. If we 
339

excluded this first acquisition and kept only the second, the MRS2-MRS1 value would be consistent with other points $\left(r^{2}=0.85, y=0.98 x+0.08\right.$, bias of 0.05$)$.

\section{Liver PDFF: After The Overfeeding Intervention}

MRS measurements indicated that the PDFF in liver increased significantly $(+1.74$, paired $t-$ test $\mathrm{p}=0.002$ ) between the two examinations. Similarly, for CSE-MRI measurements, the PDFF in liver increased significantly $(+1.35$, paired $t$-test $\mathrm{p}=0.002)$. As shown in the Figure 3a and 3c, data had a non-normal distribution (Shapiro-Wilk test $\mathrm{p}<0.001$ for MRS1; $<<0.001$ for MRS2; $\mathrm{p}<0.001$ for MRI1; $\mathrm{p}<0.001$ for MRI2) and a non-parametric test (paired sample Wilcoxon rank test) was performed (same observation: positive difference for MRS2-MRS1 $\mathrm{p}<0.001$ and positive difference for MRI2-MRI1 $\mathrm{p}<0.001)$.

\section{FA Composition Of VAT And SAT}

Some VAT spectra were not analyzed due to poor quality of spectra (low SNR or high inhomogeneity of B0 field) and were removed from the analyses: 4/20 at baseline and 3/21 after overfeeding. The CSE-MRI data of one subject could not be processed to estimate the FA composition.

\section{FA Composition: Variabilities}

For CSE-MRI measurement at the first examination, the intra-subject variability was high with a larger heterogeneity of nmidb values in VAT (mean CV of: ndb $28 \%$, nmidb $73 \%, \mathrm{n}=$ 20) than in SAT (mean CV of: ndb 23\%, nmidb 55\%). A second calculation was performed excluding pixels at the boundaries of the mask and pixels with an estimation of $\mathrm{T} 2 *$ under 10 ms and permitted to reduce the intra-subject variability (mean CV of ndb $22 \%$, nmidb $52 \% \mathrm{n}$ $=20$ for $\mathrm{VAT}$ and ndb $16 \%$, nmidb $33 \% \mathrm{n}=20$ for SAT). The mean percentage of pixels at the boundaries of the mask was $27 \%$ for SAT and 39\% for VAT. $17 \%$ pixels of SAT and $24 \%$ 
362 pixels of VAT had an estimated T2* under $10 \mathrm{~ms}$. The mean CV of MRS measurement was

363 lower in SAT (ndb 3\%, nmidb 5\%, $\mathrm{n}=20)$ than in VAT (ndb 11\%, nmidb 21\%, $\mathrm{n}=16$ ).

364

365

\section{FA Composition: Comparison MRS And CSE-MRI (Whole Volume)}

MRS and whole-volume CSE-MRI FA composition estimations were correlated (SAT: $r=$ 0.82, $\mathrm{p}<0.001$ for $\mathrm{ndb}$ and $\mathrm{r}=0.84, \mathrm{p}<0.001$ for $\mathrm{nmidb}, \mathrm{n}=19)$ with a low bias $(0.06$ IC 95 $\%$ [-0.32 0.43] for ndb and 0.02 IC $95 \%$ [-0.15 0.20] for nmidb). It was however difficult to compare these two methods without considering the high spatial variability (intra-subject variability) of CSE-MRI measurements. As illustrated in Figure 5, the MRS value was included in the range defined by the mean \pm one standard deviation of CSE-MRI measurements for each subject.

\section{FA Composition: SAT And VAT}

Regarding CSE-MRI results from the whole segmented volume (L2 to L4) versus the localized-MRS results, the comparison of FA composition of SAT and VAT appeared to be substantially equivalent in average for ndb and nmidb measurements (CSE-MRI SAT vs VAT: $\mathrm{ndb}=2.43 \pm 0.16$ vs $2.20 \pm 0.15 \mathrm{p}<0.001, \mathrm{nmidb}=0.56 \pm 0.07$ vs $0.46 \pm 0.07 \mathrm{p}<$ $0.001 ;$ MRS: $\mathrm{ndb}=2.48 \pm 0.29$ vs $1.90 \pm 0.63 \mathrm{p}=0.001, \mathrm{nmidb}=0.58 \pm 0.14$ vs $0.37 \pm 0.22$ $\mathrm{p}=0.001$ ) and for $\mathrm{PUFA}_{\text {indx }} / \mathrm{MUFA}_{\text {indx }} / \mathrm{SFA}_{\text {indx }}$ measurements (Figure 6). In both cases, paired t-tests showed significant difference between SAT and VAT FA composition ( $\mathrm{p}<$ 0.001 for CSE-MRI measurements and $\mathrm{p}<0.01$ for MRS measurements). The inter-subject variability was higher in localized-MRS measurements than in the CSE-MRI measurements.

\section{FA Composition: Change Between The Two Examinations}

The same trend for the variations of all the FA composition parameters was observed based on both CSE-MRI and MRS measurements for VAT (figure 7). The low inter-subject variability of CSE-MRI measurement permitted to detect significant variation of adipose 
tissue composition whereas MRS measurement did not (VAT MUFA +1.22 percentage points $(\mathrm{p}<0.001)$ for CSE-MRI, +0.96 percentage points $(\mathrm{p}=0.318)$ for MRS).

\section{FA Composition: GC-MS Analysis}

Thirteen samples of SAT were analyzed using gas chromatography (7 before and 6 after the overfeeding intervention). Results were expressed as molar percentage and are presented in Table 3. Measurements by GC-MS were considered as reference.

Good correlations were found between GC-MS and CSE-MRI measurements (for ndb: $\mathrm{r}=0.84, \mathrm{y}=0.76 \mathrm{x}+0.40, \mathrm{p}=0.001$; for nmidb: $\mathrm{r}=0.66, \mathrm{y}=0.88 \mathrm{x}-0.09, \mathrm{p}=0.020)$. However CSEMRI measurements overestimated ndb (Bland-Altman bias $=0.17$ percentage points, IC $95 \%$ [0.01;0.34], ) and nmidb (Bland-Altman bias=0.16 percentage points, IC 95\% [0.04;0.27], ). Better correlations were found between GC-MS and MRS measurements (for ndb: $r=0.83$, $\mathrm{y}=0.31 \mathrm{x}+1.47, \mathrm{p}<0.001$; for nmidb: $\mathrm{r}=0.74, \mathrm{y}=0.36 \mathrm{x}+0.19, \mathrm{p}=0.006)$ however the bias was larger. MRS measurements overestimated ndb (bias=0.24 percentage points, IC 95\% [0.22;0.70], Bland-Altman) and nmidb (bias=0.19 percentage points, IC 95\% [- 0.04;0.42], Bland-Altman).

\section{DISCUSSION}

Consistent with the literature (24), we found that CSE-MRI-based fat mass measurements of android region is strongly correlated with DEXA measurements. However, a small bias was observed with slight lower value of TAT volume with CSE-MRI measurements. The DEXA measurement is a 2D measurement and does not permit to distinguish VAT, SAT and other ectopic fat like bone marrow and intramuscular adipose tissue. The automated segmentation processing based on CSE-MRI images enabled to separate VAT from SAT. Thus, muscles and bone marrow were excluded from VAT mask by a threshold empirically defined at 55\% using the PDFF mapping. This exclusion could explain the difference in CSE-MRI and 
410 DEXA measurements as when we considered the PDFF of muscles, vertebrae and VAT in the

411 calculation of fat content, the bias reduced. To go further in the segmentation of different 412 adipose tissue depots, some studies $(25,26)$ differentiated the deep (DSAT) from the 413 superficial (SSAT) subcutaneous adipose tissue in the abdominal region. This segmentation of 414 DSAT and SSAT is very challenging because the fascia superficial separating these tissues 415 appears subtle and discontinuous and need a fine resolution $(\sim 1.25 \mathrm{~mm})$. The present in-plane 416 resolution was due to a compromise between an acquisition time compatible with breath417 holding and the size of the covering volume (320 $\mathrm{mm}$ in the head-foot direction in this study) 418 to give a good SNR which is an essential parameter to estimate the FA composition (i.e. 419 proportions of $\mathrm{PUFA}_{\text {indx }}, \mathrm{MUFA}_{\text {indx }}$ and $\left.\mathrm{SFA}_{\text {indx }}\right)$. From a metabolic point of view, GC 420 analysis of SSAT and DSAT in a large cohort shows homogenous FA composition in the two 421 adipose tissue depots (7).

422 Liver PDFF results were consistent with the literature (13-15), with a good correlation 423 established between MRS and CSE-MRI measurements. However, some studies (13-15) 424 provided a better correlation. It should be noted that the range of values was larger in these papers $(0-40 \%(13-15))$ than in the present paper $(0-12 \%)$. Especially, it is challenging to 426 accurately estimate very low fat content in liver. Here, we demonstrated that even if the PDFF 427 measurement methods with CSE-MRI or MRS were different, the difference between the two 428 PDFF measurements (before and after the overfeeding) was equivalent with the two methods. 429 In this study, no T2 correction was applied on the calculation of fat content in the liver for 430 MRS measurement. In addition, MRI method include by a priori knowledge eight fat spectral 431 component in the quantification model, that was not the case for MRS since fat quantities 432 were too low in this study. These could explain why the MRS measurement gave 433 underestimated values compared to CSE-MRI measurement. On the other hand, the CSE-MRI 434 PDFF estimate could be affected by the low SNR. 
435 The lipid quantification by CSE-MRI could be interesting in longitudinal clinical studies, as

436 an indirect marker of AT metabolism. Here, the average on the whole segmented volume

437 (between L2-L4) seemed to give results consistent with the literature. Machann et al. (27)

438 reported similar results even if they computed different FA composition indexes: the 439 polyunsaturated index (PUI = diacyl-methyl ratio) and the unsaturated index (UI = olefinic440 methyl ratio), VAT was composed by $14.9 \% / 48.9 \% / 36.2 \%$ of $\mathrm{PUFA}_{\text {indx }} / \mathrm{MUFA}_{\text {indx }} / \mathrm{SFA}_{\text {indx }}$ 441 (vs $15.3 \% / 42.7 \% / 43.0 \%$ in our study), SAT was composed by $16.2 \% / 56.6 \% / 27.2 \%$ (vs $44218.6 \% / 43.7 \% / 37.7 \%$ in our study). Other studies reported higher values of nmidb both using 443 MRS method (0.75 SCAT calf (28), 0.687/0.745 for VAT/DSAT (26), 0.71 for SCAT leg 444 (11), 0.79/0.81 for VAT/DSAT (12) than 0.37/0.58 for VAT/SAT in our study) and with MRI 445 method $(0.836 / 0.936$ for VAT/SAT (10), $0.71 / 0.73$ for VAT/DSAT (12), 0.74 for SCAT leg 446 (11) than $0.46 / 0.56$ for VAT/SAT in our study) which led to an estimation of PUFA superior 447 to $20 \%$. However, several gas chromatography analysis have quantified a lower content of 448 PUFA (12.3\% (29), 14.4\% (30), 16.5\% (9), 14.1\%(31)) and lower nmidb (0.45(30), 0.50 (9), $4490.63(32))$ which was more consistent with our present results. It should also be noted that the 450 PUFA $_{\text {indx }}$ calculation made from nmidb, was not an absolute quantification but a coherent 451 index of polyunsaturation, leading to consistent results when the most frequently identified 452 PUFA is di-unsaturated FA. It could be possible to quantify the proportion of PUFA more 453 precisely by calculating the proportion of omega-3 (33-35) and correcting the present 454 calculation but it does not permit to know exactly the number of double bonds per chain.

455 The validation of lipid quantification by CSE-MRI using MRS measurement is a difficult 456 task. As expected, the lipid quantification by MRS is challenging and results depend on the 457 B0 field heterogeneities (26), the MRS sequences (36), the acquisition parameters (long TE or 458 short TE) (29) and the fitting approaches (37). It is therefore difficult to use it as reference. On 459 the other hand, a gold standard like gas chromatography-mass spectrometry analysis is 
460 difficult to perform on human volunteers, for ethical reasons only subcutaneous adipose tissue 461 can be analyzed. In this study, good correlations were found between MRS and CSE-MRI measurement, MRS and GC-MS measurements and between CSE-MRI and GC-MS 463 measurements. However, the NMR methodologies gave biased results compare to GC-MS 464 methodology. The CSE-MRI and MRS fitting approach used strong constraint on a relation 465 between ndb and nmidb $(10,19)$ to improve the repeatability of measurements, however this 466 constraint provided biased results and not absolute quantification. Therefore, the good 467 correlation between GC-MS and NMR methodologies is already an important result, even if, a comparative study using more sample should be necessary. The higher inter-subject variability seen in localized-MRS measurements compared with the CSE-MRI measurements 470 could be explained first by the difficulty to locate in some lean volunteers, the MRS voxel 471 into AT only. This could induce some partial volume effects. Moreover, due to the large 472 difference between the elementary volume ( $8000 \mathrm{~mm} 3$ for MRS compared to $9.84 \mathrm{~mm} 3$ for 473 MRI), the local inhomogeneity is in favor of CSE-MRI despite a dedicated localized shim for 474 MRS. Finally, the value estimated by CSE-MRI resulted from an averaging over a large 475 number of pixels (>10000 pixels) which attenuated the effect of potential aberrant individual 476 values for each subjects, unlike for the MRS where only two measurements were made. The 477 spatial non-uniformity of nmidb was larger in VAT than in SAT. This high spatial variability 478 could be due to a sensitivity of CSE-MRI FA composition measurements to local field 479 inhomogeneity as the coefficient of variation reduced when we removed pixels located at fat480 air interface. This local field inhomogeneity is due to high contribution of internal field as 481 regard to fat-tissue interface where susceptibility gradient is strong. To increase spatial 482 resolution may probably minimize this effect. Alternatively, to provide more consistent 483 measurements, it is possible to suppress these voxels in the segmentation pipeline such as 484 proposed in this study. Moreover, VAT tissue could be more impacted by local field 
485 inhomogeneity due to the presence of air in the gut. In localized area, this sensitivity to local 486 field heterogeneities led to erroneous values of CSE-MRI measurements. For the study of 487 localized area, localized-MRS seemed to still be the best method thanks to shim methods 488 allowing to homogenize locally the magnetic field. Visual analysis of spectrum permits to 489 validate results with consistent fitting approach. Moreover, in the literature, only methods of 490 spectroscopy make the quantification of omega-3 possible (33-35). A limitation of our study 491 concerned the design of the overfeeding. This was a short term 31-days overfeeding, and as 492 the half-life of subcutaneous adipose tissue fatty acids is around 6 to 9 months (9), the 493 overfeeding may have been too short to detect a change in SAT fatty composition with the 494 diet.

495 To conclude, significant increase in fat distribution in VAT, SAT and liver were measured by 496 DEXA, MRS and CSE-MRI measurements after an experimental overfeeding. FA 497 composition changes (more precisely the MUFA proportion) of the adipose tissue were also 498 observed by CSE-MRI measurements. The use of a single 3D CSE-MRI sequence associated 499 with a dedicated post processing method is a suitable protocol to follow several parameters 500 linked to adipose tissue changes. This NMR protocol could be used in future work to evaluate 501 non-invasively the impact of nutrition on adipose tissues and liver. 


\section{Acknowledgments:}

504 This study was conducted as part of the LABEX PRIMES (ANR-11-LABX-0063) of the

505 "Université de Lyon", within the "Investissements d'Avenir" program (ANR-11-IDEX-0007)

506 operated by the French National Research Agency (ANR). This study was also supported by 507 the IHU OpéRa (ANR-10-IBHU-0004), within the "Investissements d'Avenir"program 508 operated by the French National Research Agency (ANR). We thank Corinne Louche509 Pelissier and Adeline Cestre (CRNH-RA) for technical assistance in the fatty acid analyse 510 using GC-MS. 


\section{References}

512 1. Heymsfield SB, Wadden TA: Mechanisms, Pathophysiology, and 513 Management of Obesity. N Engl J Med 2017; 376:254-266.

514 2. Lee JJ, Pedley A, Hoffmann U, Massaro JM, Levy D, Long MT: Visceral and 515 Intrahepatic Fat Are Associated with Cardiometabolic Risk Factors Above Other 516 Ectopic Fat Depots: The Framingham Heart Study. Am J Med 2018; 131:684517 692.e12.

518 3. Alligier M, Gabert L, Meugnier E, et al.: Visceral Fat Accumulation During 519 Lipid Overfeeding Is Related to Subcutaneous Adipose Tissue Characteristics in 520 Healthy Men. J Clin Endocrinol Metab 2013; 98:802-810.

4. Chowdhury B, Sjöström L, Alpsten M, Kostanty J, Kvist H, Löfgren R: A multicompartment body composition technique based on computerized tomography. Int J Obes Relat Metab Disord J Int Assoc Study Obes 1994; 18:219-234.

5. $\mathrm{Hu} \mathrm{HH}$, Kan HE: Quantitative proton MR techniques for measuring fat. NMR Biomed 2013; 26:1609-1629.

6. Reeder SB, Hu HH, Sirlin CB: Proton density fat-fraction: A standardized mrbased biomarker of tissue fat concentration. J Magn Reson Imaging 2012; 36:1011-1014.

7. Petrus P, Edholm D, Rosqvist F, et al.: Depot-specific differences in fatty acid composition and distinct associations with lipogenic gene expression in abdominal adipose tissue of obese women. Int J Obes 2017; 41:1295-1298.

8. Jové M, Moreno-Navarrete JM, Pamplona R, Ricart W, Portero-Otín M, 534 Fernández-Real JM: Human omental and subcutaneous adipose tissue exhibit specific lipidomic signatures. FASEB J 2013; 28:1071-1081.

536 9. Hodson L, Skeaff CM, Fielding BA: Fatty acid composition of adipose tissue and blood in humans and its use as a biomarker of dietary intake. Prog Lipid Res 2008; 47:348-380. of the triglyceride fatty acid composition with 3.0 T MRI. NMR Biomed 2014; 27:1211-1221.

542 11. Peterson $\mathrm{P}$, Månsson S: Simultaneous quantification of fat content and fatty acid composition using MR imaging. Magn Reson Med 2013; 69:688-697. 
544 12. Bydder M, Girard O, Hamilton G: Mapping the double bonds in 545 triglycerides. Magn Reson Imaging 2011; 29:1041-1046.

546 13. Achmad E, Yokoo T, Hamilton G, et al.: Feasibility of and agreement 547 between MR imaging and spectroscopic estimation of hepatic proton density fat 548 fraction in children with known or suspected nonalcoholic fatty liver disease. 549 Abdom Imaging 2015; 40:3084-3090.

550 14. Zand KA, Shah A, Heba E, et al.: Accuracy of multiecho magnitude-based 551 MRI (M-MRI) for estimation of hepatic proton density fat fraction (PDFF) in 552 children. J Magn Reson Imaging 2015; 42:1223-1232.

553 15. Tyagi A, Yeganeh $\mathrm{O}$, Levin $\mathrm{Y}$, et al.: Intra- and inter-examination 554 repeatability of magnetic resonance spectroscopy, magnitude-based MRI, and 555 complex-based MRI for estimation of hepatic proton density fat fraction in 556 overweight and obese children and adults. Abdom Imaging 2015; 40:3070-3077.

557 16. Stults-Kolehmainen MA, Stanforth PR, Bartholomew JB, Lu T, Abolt CJ, 558 Sinha R: DXA estimates of fat in abdominal, trunk and hip regions varies by 559 ethnicity in men. Nutr Diabetes 2013; 3:e64.

560 17. Folch J, Lees M, Sloane Stanley GH: A simple method for the isolation and 561 purification of total lipides from animal tissues. J Biol Chem 1957; 226:497509.

563

564

565

566

567

568

569

570

571

572

573

574

575

576

577

578

18. Gabert L, Vors C, Louche-Pélissier C, et al.: 13C tracer recovery in human stools after digestion of a fat-rich meal labelled with [1,1,1-13C3]tripalmitin and [1,1,1-13C3]triolein. Rapid Commun Mass Spectrom ; 25:2697-2703.

19. Nemeth A, Segrestin B, Leporq B, et al.: Comparison of MRI-derived vs. traditional estimations of fatty acid composition from MR spectroscopy signals. NMR Biomed 2018; 31:e3991.

20. Ratiney H, Bucur A, Sdika M, Beuf O, Pilleul F, Cavassila S: Effective voigt model estimation using multiple random starting values and parameter bounds settings for in vivo hepatic $1 \mathrm{H}$ magnetic resonance spectroscopic data. In 2008 5th IEEE Int Symp Biomed Imaging Nano Macro; 2008:1529-1532.

21. Leporq B, Ratiney H, Pilleul F, Beuf O: Liver fat volume fraction quantification with fat and water $\mathrm{T} 1$ and $\mathrm{T} 2 *$ estimation and accounting for NMR multiple components in patients with chronic liver disease at 1.5 and 3.0 T. Eur Radiol 2013; 23:2175-2186.

22. Lankton S, Tannenbaum A: Localizing Region-Based Active Contours. IEEE Trans Image Process 2008; 17:2029-2039. 
579

580

581

582

583

584

585

586

587

588

589

590

591

592

593

594

595

596

597

598

599

600

601

602

603

604

605

606

607

608

609

610

611

612

613

23. Snyder W, Cook M, Nasset E, Karhausen L, Parry Howells G, Tipton I: Report of the Task Group on Reference Man. International Commission on radiological protection, no. 23. Pergamon Press: Oxford; 1975.

24. Silver HJ, Niswender KD, Kullberg J, et al.: Comparison of Gross Body FatWater Magnetic Resonance Imaging at 3 Tesla to Dual Energy X-Ray Absorptiometry in Obese Women. Obes Silver Spring Md 2013; 21:765.

25. Sadananthan SA, Prakash B, Leow MK-S, et al.: Automated segmentation of visceral and subcutaneous (deep and superficial) adipose tissues in normal and overweight men. J Magn Reson Imaging 2015; 41:924-934.

26. Hamilton G, Schlein AN, Middleton MS, et al.: In vivo triglyceride composition of abdominal adipose tissue measured by $1 \mathrm{H}$ MRS at 3T. J Magn Reson Imaging 2017; 45:1455-1463.

27. Machann J, Stefan N, Wagner R, et al.: Intra- and interindividual variability of fatty acid unsaturation in six different human adipose tissue compartments assessed by 1H-MRS in vivo at 3 T. NMR Biomed 2017; 30:e3744.

28. Ren J, Dimitrov I, Sherry AD, Malloy CR: Composition of adipose tissue and marrow fat in humans by $1 \mathrm{H}$ NMR at 7 Tesla. J Lipid Res 2008; 49:20552062.

29. Lundbom J, Hakkarainen A, Fielding B, et al.: Characterizing human adipose tissue lipids by long echo time $1 \mathrm{H}-\mathrm{MRS}$ in vivo at 1.5 Tesla: validation by gas chromatography. NMR Biomed 2010; 23:466-472.

30. Field CJ, Angel A, Clandinin MT: Relationship of diet to the fatty acid composition of human adipose tissue structural and stored lipids. Am J Clin Nutr 1985; 42:1206-1220.

31. Rosqvist F, Iggman D, Kullberg J, et al.: Overfeeding Polyunsaturated and Saturated Fat Causes Distinct Effects on Liver and Visceral Fat Accumulation in Humans. Diabetes 2014; 63:2356-2368.

32. Garaulet M, Hernandez-Morante JJ, Lujan J, Tebar FJ, Zamora S: Relationship between fat cell size and number and fatty acid composition in adipose tissue from different fat depots in overweight/obese humans. Int J Obes 2006; 30:899-905.

33. Lundbom J, Heikkinen S, Fielding B, Hakkarainen A, Taskinen M-R, Lundbom N: PRESS echo time behavior of triglyceride resonances at 1.5T: Detecting $\omega-3$ fatty acids in adipose tissue in vivo. J Magn Reson 2009; $201: 39-$ 47. 
614 34. Fallone CJ, McKay RT, Yahya A: Long TE STEAM and PRESS for 615 estimating fat olefinic/methyl ratios and relative $\omega-3$ fat content at 3T. $J$ Magn 616 Reson Imaging 2018; 48:169-177.

617 35. Škoch A, Tošner Z, Hájek M: The in vivo J-difference editing MEGA618 PRESS technique for the detection of n-3 fatty acids. NMR Biomed 2014; 619 27:1293-1299.

620 36. Hamilton G, Middleton MS, Bydder M, et al.: Effect of PRESS and STEAM 621 sequences on magnetic resonance spectroscopic liver fat quantification. J Magn 622 Reson Imaging 2009; 30:145-152.

623 37. Mosconi E, Sima DM, Osorio Garcia MI, et al.: Different quantification 624 algorithms may lead to different results: a comparison using proton MRS lipid 625 signals. NMR Biomed 2014; 27:431-443. 


\section{Tables}

\section{Table 1. Parameters conditioning the fat spectrum model used}

\begin{tabular}{|c|c|c|c|c|}
\hline $\begin{array}{l}\text { Component } \\
\text { k }\end{array}$ & Type & $\begin{array}{l}\Delta \mathrm{f}_{\mathrm{k}} \\
(\mathrm{Hz})\end{array}$ & $\begin{array}{l}\text { Chemical } \\
\text { shift (ppm) }\end{array}$ & $\mathrm{n}_{\mathrm{k}}(\mathrm{ndb}, \mathrm{nmidb}, \mathrm{CL})$ \\
\hline 1 & $\begin{array}{l}\text { Olefinic } \\
+ \text { Glycerol }\end{array}$ & -74.9 & $\begin{array}{l}5.29 \\
5.19\end{array}$ & $\begin{array}{l}2 \times \mathrm{ndb} \\
+1\end{array}$ \\
\hline water & - & 0 & 4.70 & $\mathrm{n}_{\text {water }}=2$ \\
\hline 2 & Glycerol & 63.5 & 4.20 & 4 \\
\hline 3 & Dyacil & 247.7 & 2.75 & $2 \times$ nmidb \\
\hline 4 & $\alpha$-carboxyl & 312.4 & 2.24 & 6 \\
\hline 5 & $\alpha$-olefinic & 340.4 & 2.02 & $4 \times$ (ndb-nmidb) \\
\hline 6 & $\beta$-carboxyl & 393.7 & 1.60 & 6 \\
\hline 7 & Methylene & 431.8 & 1.30 & $6 \times(C L-4)-8 \times n d b+2 \times n m i d b$ \\
\hline 8 & Methyl & 482.6 & 0.90 & 9 \\
\hline
\end{tabular}

629

Table 1: Parameters conditioning the fat spectrum model used. $\Delta \mathbf{f}_{\mathrm{k}}$, frequency shift between water and each fat

630 resonance. 
Baseline

Age

Body weight $(\mathrm{kg})$

Body Mass index $\left(\mathrm{kg} / \mathrm{m}^{2}\right)$

DEXA

fat mass of whole body $(\mathrm{kg})$

fat mass of android region $(\mathrm{kg})$

CSE-MRI

SAT volume $\left(\mathrm{cm}^{3}\right)$

VAT volume $\left(\mathrm{cm}^{3}\right)$

TAT volume $\left(\mathrm{cm}^{3}\right)$

VAT/SAT

liver PDFF

localized liver PDFF
$19.6 \pm 1.2$

$1.85 \pm 0.13$

$33 \pm 2$

$79.6 \pm 1.6$

$82.4 \pm 1.6$

$26.0 \pm 0.3$

$21.2 \pm 1.1$

$2.07 \pm 0.57$

$1191 \pm 79$

$1070 \pm 116$

$2261 \pm 765$

$0,91 \pm 0,09$

$4.05 \pm 0.87$

$3.38 \pm 0.77$
Differences

$2.6 \pm 0.3 * * *(\mathrm{n}=21)$

$0.8 \pm 0.1 * * *(\mathrm{n}=21)$

$1.6 \pm 1.1 * * *(\mathrm{n}=21)$

$0.19 \pm 0.04 * *(\mathrm{n}=20, \mathrm{p}=0.001)$

$91 \pm 22 * * *(\mathrm{n}=20)$

$129 \pm 31 * * *(\mathrm{n}=20)$

$219 \pm 41 * * *(\mathrm{n}=20)$

$0,06 \pm 0,03 *(n=20, p=0.020)$

$1.35 \pm 0.39 * *(\mathrm{n}=20, \mathrm{p}=0.003)$

$1.66 \pm 0.53 * *(n=19, p=0.006)$ 
FA composition of SAT

FA composition of VAT

$$
\begin{array}{ll}
\mathrm{ndb}=2.43 \pm 0.04 & 2.43 \pm 0.03 \\
\mathrm{nmidb}=0.56 \pm 0.02 & 0.55 \pm 0.01 \\
\mathrm{ndb}=2.20 \pm 0.03 & 2.19 \pm 0.03 \\
\mathrm{nmidb}=0.46 \pm 0.02 & 0.44 \pm 0.01
\end{array}
$$

\section{$1.20 \pm 0.23$}

$\mathrm{ndb}=2.48 \pm 0.06$

$\mathrm{nmidb}=0.58 \pm 0.03$

$\mathrm{ndb}=1.90 \pm 0.16$

$\mathrm{nmidb}=0.37 \pm 0.06$
$3.02 \pm 0.31$

$2.45 \pm 0.07$

$0.57 \pm 0.03$

$2.04 \pm 0.11$

$0.41 \pm 0.04$

$$
\begin{aligned}
& 0.00 \pm 0.01(\mathrm{n}=19, \mathrm{p}=0.812) \\
& 0.00 \pm 0.01(\mathrm{n}=19, \mathrm{p}=0.612) \\
& -0.01 \pm 0.02(\mathrm{n}=19, \mathrm{p}=0.512) \\
& -0.03 \pm 0.01 *(\mathrm{n}=19, \mathrm{p}=0.013)
\end{aligned}
$$

$1.74 \pm 0.48 * *(n=20, p=0.002)$

$-0.05 \pm 0.05(\mathrm{n}=20, \mathrm{p}=0.381)$

$-0.02 \pm 0.03(n=20, p=0.417)$

$0.19 \pm 0.17(n=15, p=0.290)$

$0.06 \pm 0.06(n=15, p=0.395)$

632

Table 2: Measurements of the cohort before and after overfeeding. Data are expressed as mean SEM. * indicate significant difference due to overfeeding paired t-test $* * * p<0.001$. 


\begin{tabular}{|c|c|c|c|}
\hline & Fatty acid & Mean & SD \\
\hline \multirow[t]{6}{*}{ SFA (\%) } & C12 & 0.80 & 0.17 \\
\hline & C14 & 4.99 & 0.48 \\
\hline & C15 & 0.47 & 0.03 \\
\hline & C16 & 27.40 & 1.31 \\
\hline & C18 & 4.32 & 0.63 \\
\hline & $\mathrm{C} 20$ & 0.12 & 0.02 \\
\hline \multirow{6}{*}{$\begin{array}{l}\text { MUFA } \\
(\%)\end{array}$} & C14:1 & 0.49 & 0.12 \\
\hline & C16:1 & 4.46 & 1.14 \\
\hline & $\mathrm{C} 18: \ln 9 \mathrm{t}$ & 0.47 & 0.08 \\
\hline & C18:1n9c & 42.15 & 1.3 \\
\hline & $\mathrm{C} 18: \ln 7$ & 1.75 & 0.09 \\
\hline & C20:1 & 0.45 & 0.03 \\
\hline \multirow[t]{4}{*}{ PUFA (\%) } & C18:2n6c & 11.09 & 2.32 \\
\hline & $\mathrm{C} 20: 2$ & 0.15 & 0.01 \\
\hline & $\mathrm{C} 18: 3 \mathrm{n} 3$ & 0.68 & 0.18 \\
\hline & C20:4n6 & 0.20 & 0.05 \\
\hline \multicolumn{2}{|l|}{ PUFA (\%) } & 12.12 & 2.44 \\
\hline \multicolumn{2}{|l|}{ MUFA $(\%)$} & 499.78 & 2.27 \\
\hline \multicolumn{2}{|l|}{ SFA (\%) } & 38.10 & 1.98 \\
\hline \multicolumn{2}{|l|}{$\overline{\mathbf{n d b}_{\mathrm{GC}}}$} & 2.25 & 0.12 \\
\hline \multicolumn{2}{|l|}{ nmidb $_{\mathrm{GC}}$} & 0.40 & 0.08 \\
\hline \multicolumn{2}{|l|}{$\mathbf{C L}_{\mathrm{GC}}$} & 17.10 & 0.06 \\
\hline \multicolumn{2}{|c|}{$\operatorname{PUFA}_{\text {indx }}(\%)$} & 13.21 & 2.57 \\
\hline \multicolumn{2}{|c|}{ MUFA $_{\text {indx }}(\%)$} & 48.69 & 2.37 \\
\hline \multicolumn{2}{|l|}{ SFA $_{\text {indx }}(\%)$} & 38.10 & 1.98 \\
\hline
\end{tabular}




\section{$641 \quad$ Figure Legends}

642

643

644

645

646

647

648

649

650

652

653

654

655

656

657

658

659

660

661

662

663

Figure 1: A STEAM sequence was acquired in liver, subcutaneous adipose tissue (SAT) and visceral adipose tissue (VAT) using the following parameters: TR $=3000 \mathrm{~ms}$, $\mathrm{TE}=14 \mathrm{~ms}, \mathrm{TM}=10 \mathrm{~ms}$, VOI of $20 \times 20 \times 20 \mathrm{~mm} 3$, no water suppression, $2048 \mathrm{~Hz}$ bandwidth, 1024 data points, 4 signal averages for VAT and SAT and 32 signal averages for the liver. For SAT and VAT, further acquisitions were made with $\mathrm{TE}=[24,34,44,54,64] \mathrm{ms}$.

Figure 2: The total abdominal adipose tissue (TAT) volume delimited by the lumbar vertebrae L2 and L4 was calculated using CSE-MRI. This volume was converted into mass using the assumed density of $0.92 \mathrm{~g} / \mathrm{cm} 3$ and compared to the DEXA measurement on android region. The dotted line in the left part of this graph represents the identity line.

Figure 3: Dots represent the value of the proton density fat fraction (PDFF) in the liver for each subject and are represented in a boxplot beside dots. Figures a) and b) show CSEMRI measurements. Figures c) and d) show MRS measurements. In figures a) and c) data before overfeeding (MRI1 and MRS1) are in pink color and data after the overfeeding (MRI2 and MRS2) in blue. In figures b) and d) the difference between the two examinations was illustrated. Paired t-test was performed between MRI1 and MRI2 for the PDFF in the liver. Significant difference between the two examinations was shown by Paired t-test $(* *$ p-value < $0.01)$ and Wilcoxon rank test $(\mathrm{p}<0.001), \mathrm{n}=20$, . The PDFF increased significantly $(+1.35, \mathrm{p}$ $=0.002$ for CSE-MRI measurements; $+1.74, \mathrm{p}=0.002$ for MRS measurements). Figure 4: The left part of this graph shows a good correlation between $\Delta \mathrm{MRI}$ (localized MRI2-MRI1 measurement) and $\triangle$ MRS (MRS2-MRS1 measurement). The Pearson's coefficient was $r=0.88, \mathrm{p}<0.001$. The Bland-Altman in the right part of the graph shows a little bias of 0.05 . 
Figure 5: For each subject $(\mathrm{n}=21$, baseline examination, abdominal subcutaneous

665 adipose tissue), the mean value of ndb (a) and nmidb

(b) is in red color for CSE-MRI measurement and in black for MRS measurement. The error bars represent the standard deviation in the whole volume studied for CSE-MRI measurements and the mean deviation 668 between the MRS test-retest measurements. The CSE-MRI measurements of subject ' 1 ' were missing. The MRS measurements of subject '20' were missing.

Figure 6: Bars represent the mean value of PUFAindx (resp. MUFAindx and 671 SFAindx) and error bars represent the standard deviation (inter-subject variability) for the 672 tissue VAT (visceral adipose tissue) and SAT (abdominal subcutaneous adipose tissue) measured a) by MRS and b) by CSE-MRI method. Paired t-tests were performed between 674 values from SAT against values from VAT for PUFAindx, MUFAindx and SFAindx 675 measurements. $* * * \mathrm{p}<0.001, * * \mathrm{p}<0.01$

Figure 7: Dots represent the difference between the baseline measurement and after

677 the overfeeding. The inter-subject of MRS measurement was higher than CSE-MRI 678 measurement. Paired t-tests were performed between the two examinations (SAT: 679 PUFAindx/MUFAindx/SFAindx $-0.12(\mathrm{p}=0.356) / 0.34(\mathrm{p}=0.001) /-0.23(\mathrm{p}=0.356) \mathrm{n}=19$ 680 for CSE-MRI and -0.94 $(\mathrm{p}=0.416) /-0.20(\mathrm{p}=0.314) / 1.15(\mathrm{p}=0.354) \mathrm{n}=18$ for MRS; VAT: 681 682 $-0.84(\mathrm{p}=0.012) / 1.22(\mathrm{p}<0.001) /-0.38(\mathrm{p}=0.349) \mathrm{n}=19$ for CSE-MRI and $-0.38(\mathrm{p}=$ $0.604) / 0.96(p=0.318) /-0.58(p=0.443) n=13$ for MRS $)$. 


\section{Voxel location}
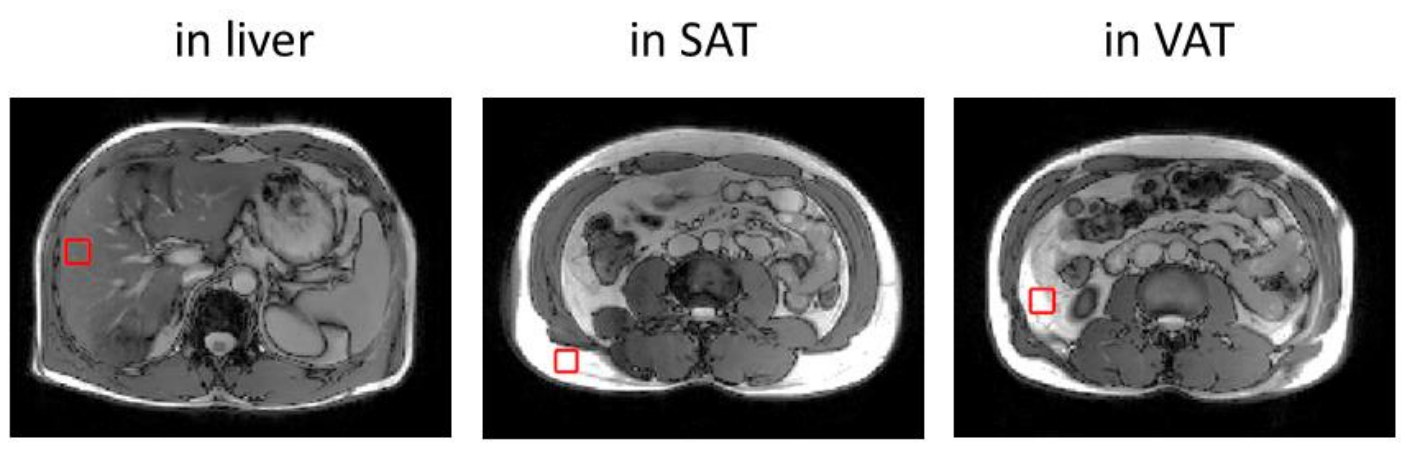

\section{Associated spectum}

684
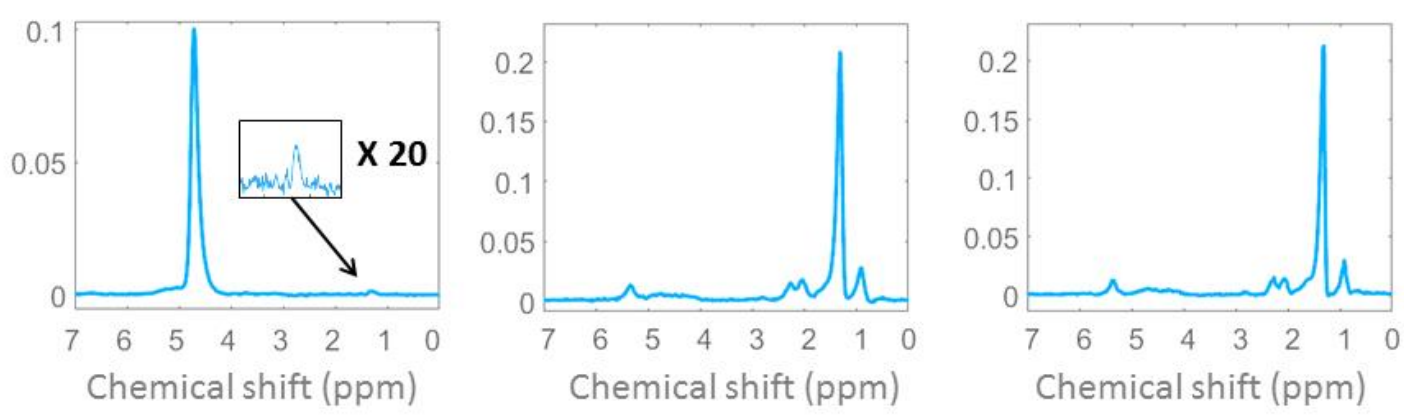

MRI vs DEXA
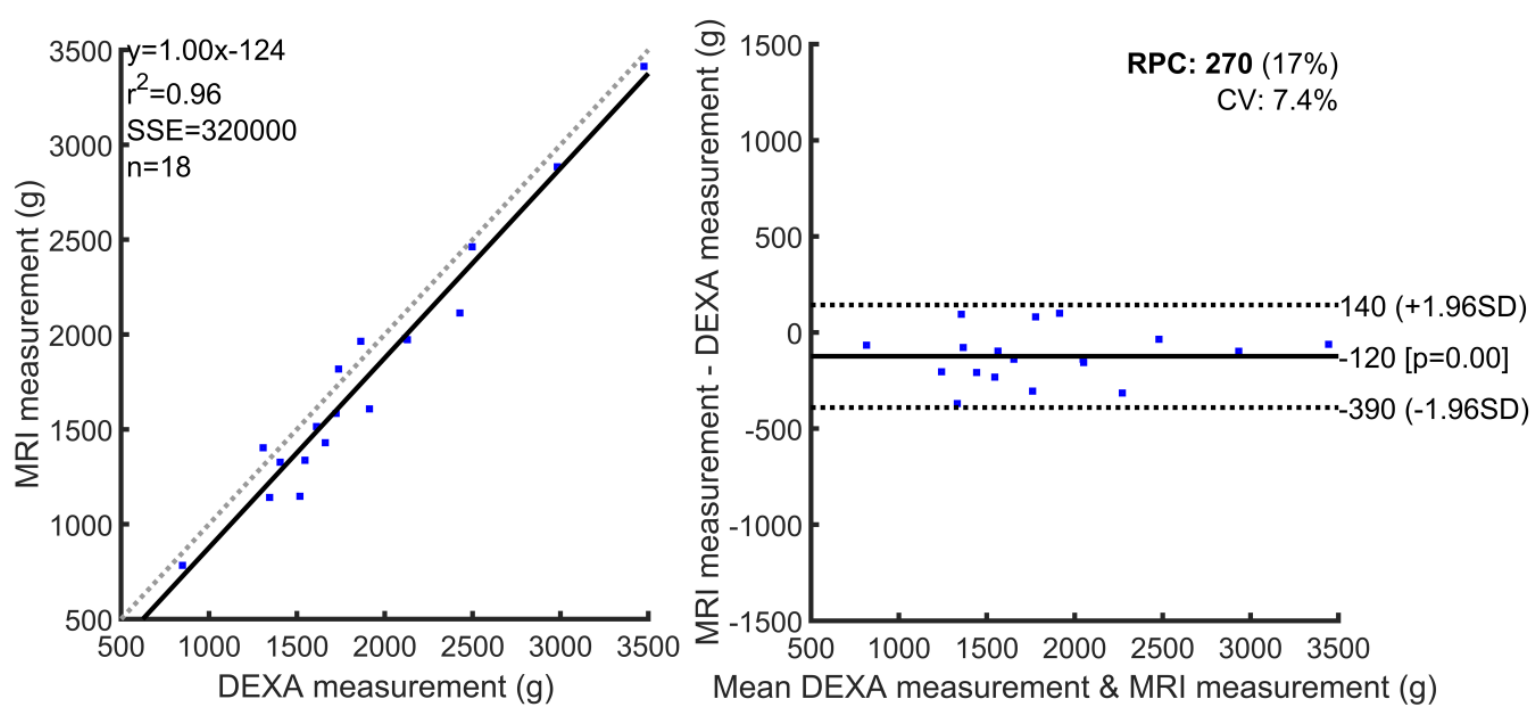
a)

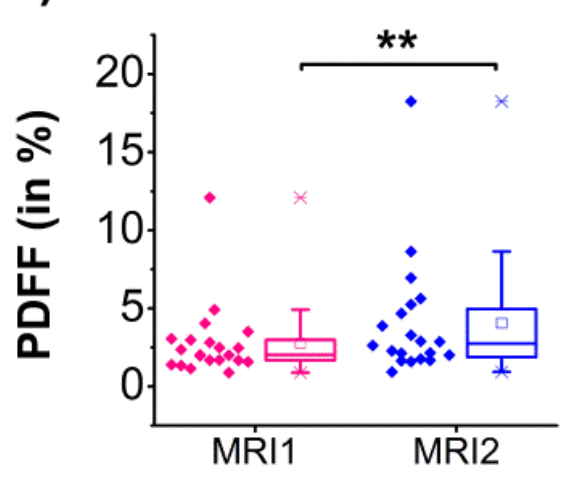

c)

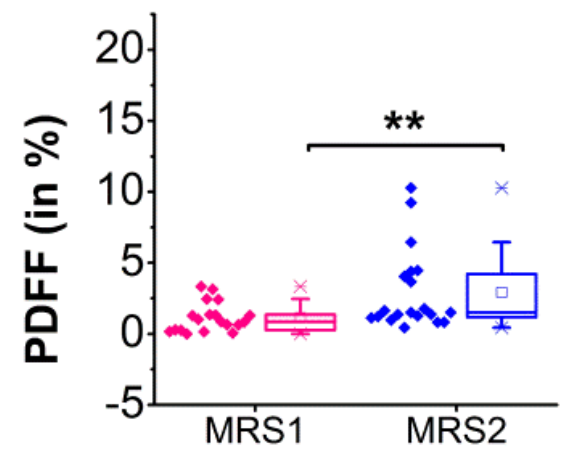

b)

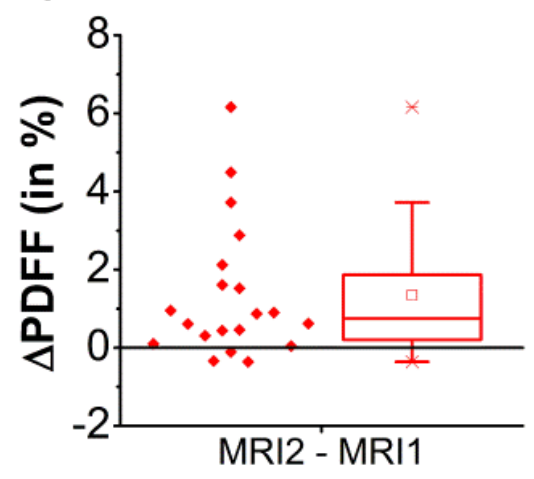

d)

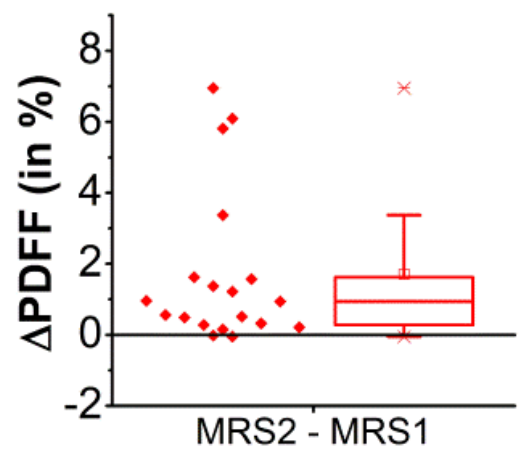

688

689 Figure 3

$\triangle$ PDFF (in \%) in liver
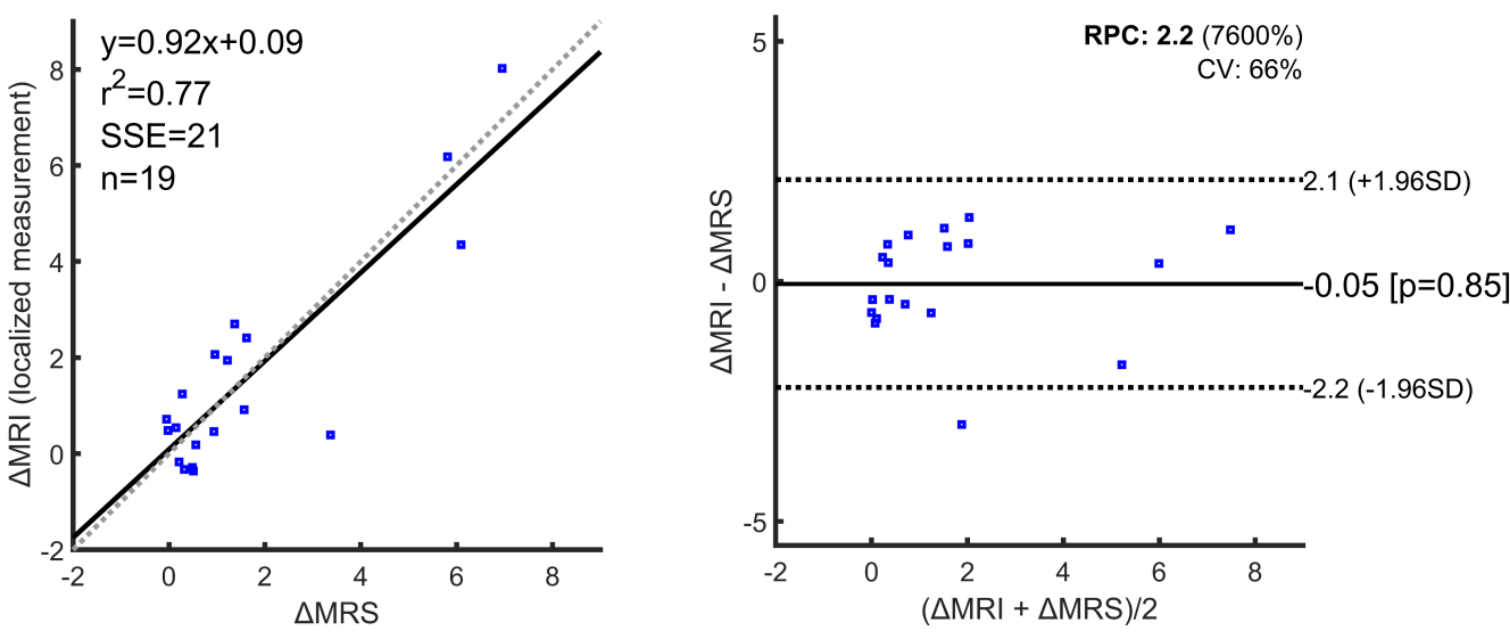

690

$691 \quad$ Figure 4 
a)

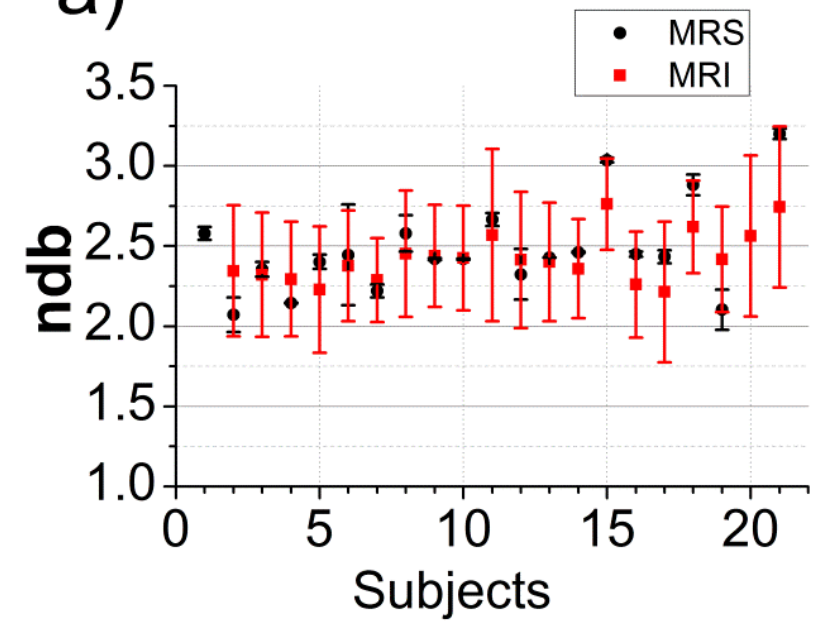

692 b)

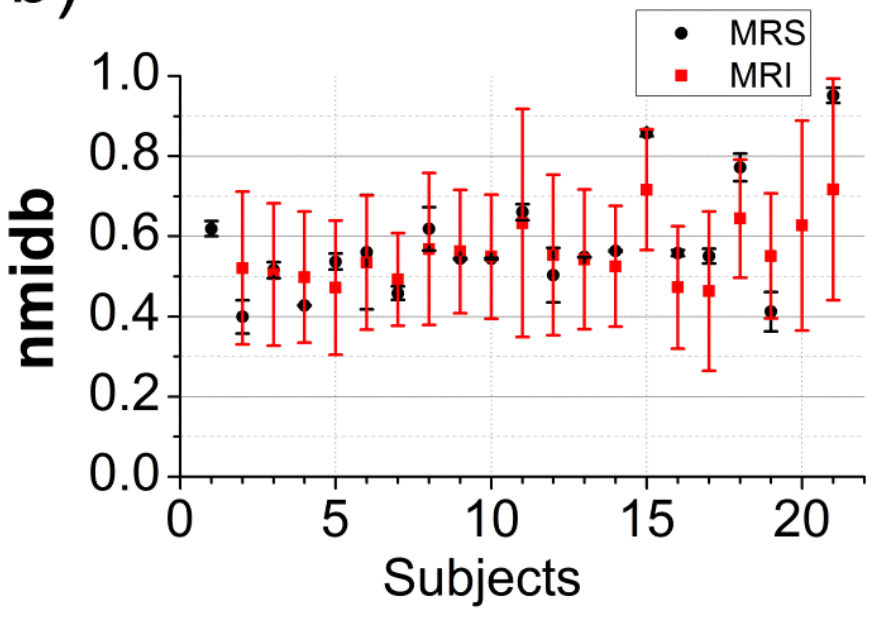

693 Figure 5

a)

MRS measurements

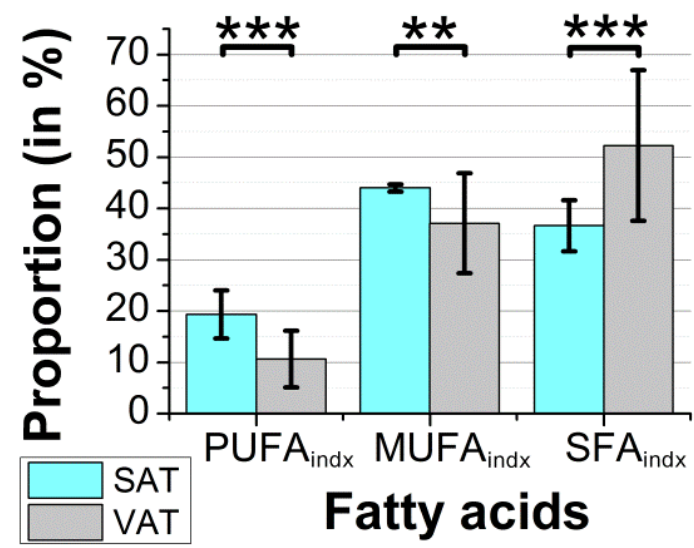

b)

MRI measurements

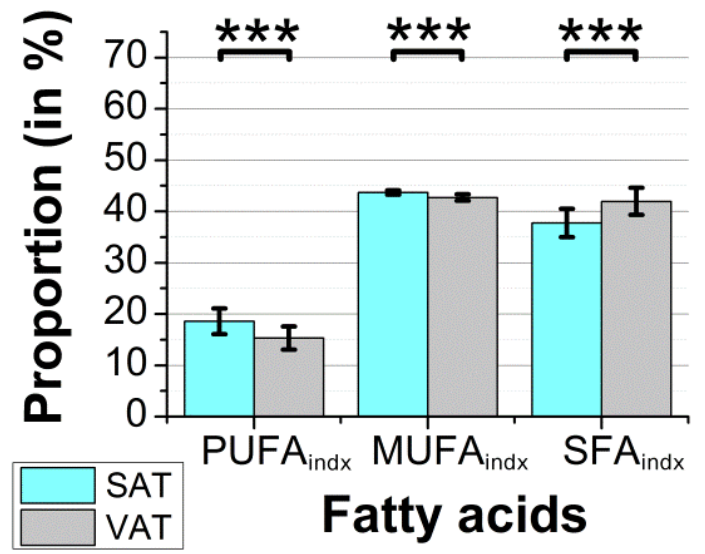

694 

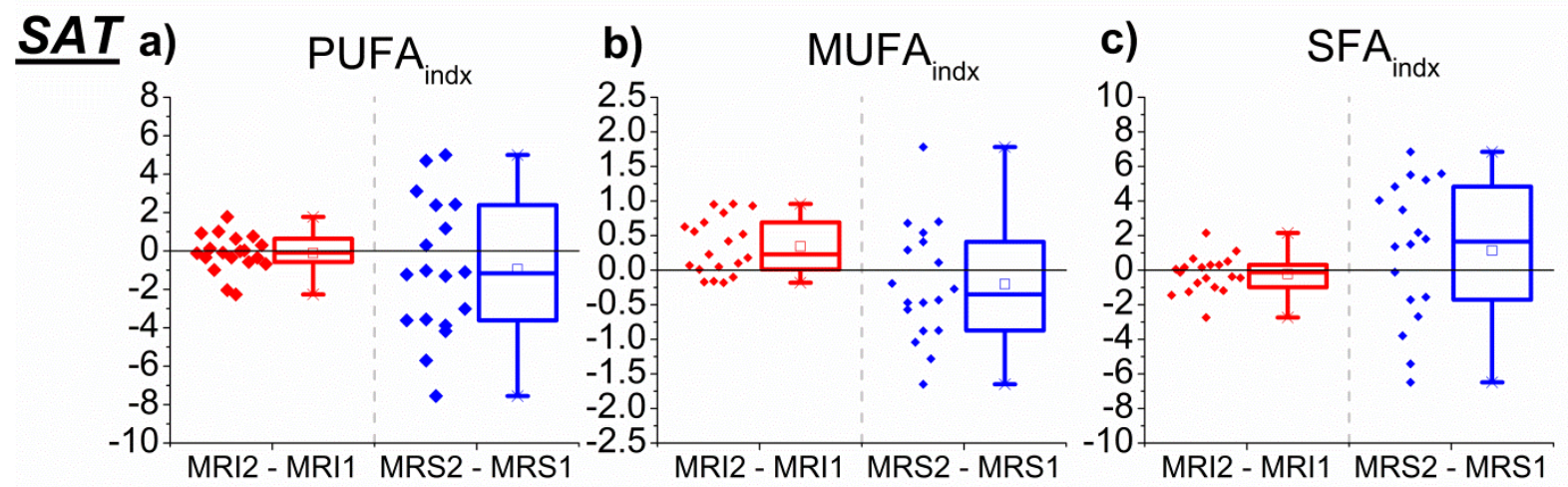

VAT

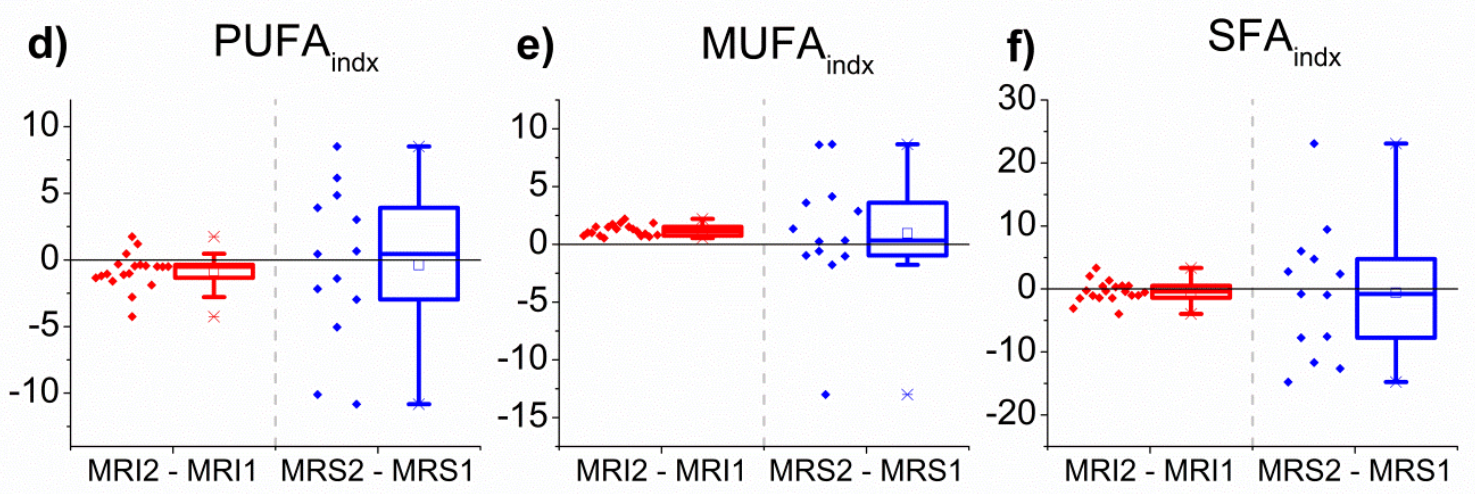

696

697 Figure 7 


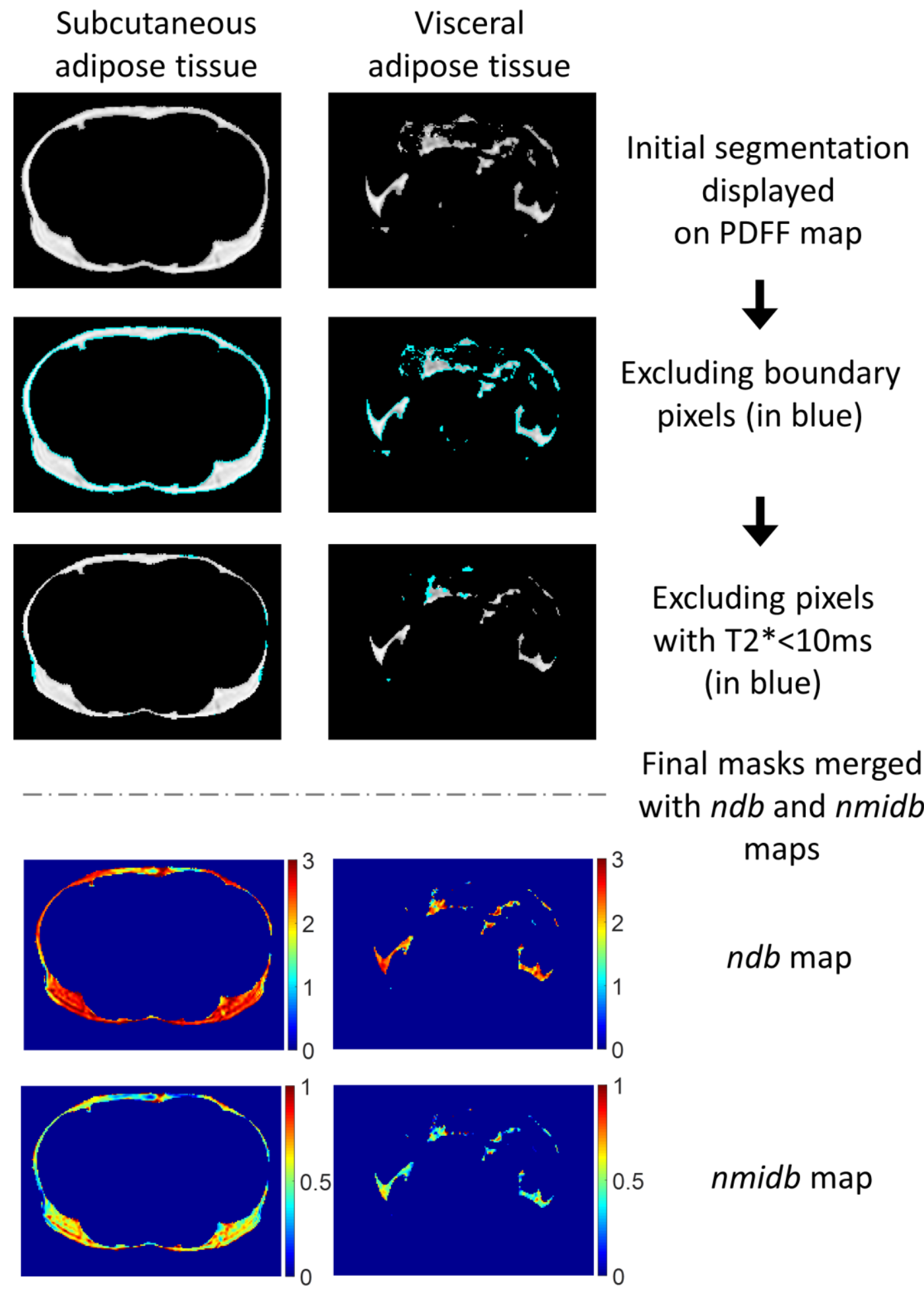

Supplementary figure 1: Procedure of pixel exclusion after the initial segmentation to compute the mean ndb (number of double bonds) and nmidb (number of methylene-interrupted double bonds). Pictures in grey scale are displaying the subcutaneous and visceral adipose tissue masks merged with the PDFF maps. Pictures in color scale are displaying the subcutaneous and visceral adipose tissue masks merged with the ndb and nmidb maps. 


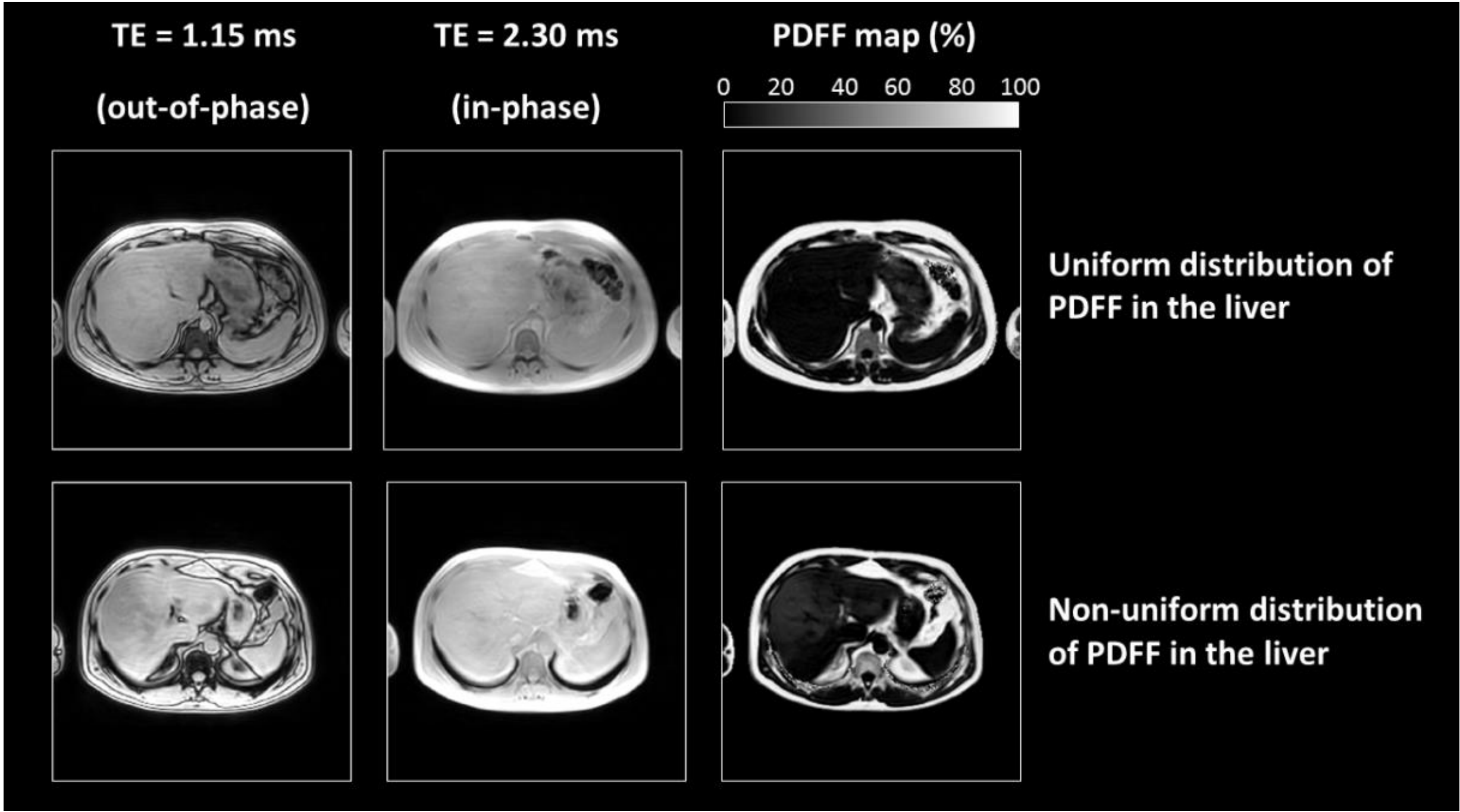

Supplementary figure 2: Two examples of exams with different PDFF distribution in the liver. 3D MRI acquisition parameters were the following : 8 echoes ( $\mathrm{n} \times 1.15 \mathrm{~ms}$ TE with $\mathrm{n}=1, \ldots, 8), 5^{\circ}$ flip angle, $10.3 \mathrm{~ms}$ TR, $384 \times 420 \times 320 \mathrm{~mm} 3 \mathrm{FOV}, 256 \times 256 \times 80$ matrix size, $20.8 \mathrm{~s}$ scan time. This acquisition was made in breath-holding. The first (out of phase) and second (in-phase) echoes as well as the PDFF map are displayed. 